\title{
Research, study, design and development of an artificial ear splint model by using a 3D printer
}

\author{
Athanasios Argyropoulos ${ }^{1, *}$, Pantelis N. Botsaris ${ }^{1}$ \\ ${ }^{1}$ Democritus University of Thrace, Dep. Of Production Engineering and Management, Lab. of Mechanical Design, Greece
}

\begin{abstract}
The aim of this research study centers around the design and development, using a threedimensional model of an ear-splint. This study results from the need of a non-invasive method, as the current techniques used in otoplasty have succeeded in reducing the risk of complications, but without drastically reducing the rate of postoperative deformity. Indeed, stick out ears present a deformity that occurs in 5\% of the population. Factors such as psychosocial impact and intimidation lay the foundations for the need for such a method. This paper essays to create, through 3D printing, a case customized into the ear of any person of all ages. The splint was made on Zortrax M200 3D printer model, equipped with the V3 Hotend head, and the material used for printing was 16 grams of Z-FLEX. It is noted that the creation of the case is innovative and original. After putting the ear-splint into test, by applying it on a human ear, two conclusions emerge. First, it is very light, weight, just 16 grams, and second, it offers comfort and convenience to the user. In order to get results for its practicality, a clinical rehabilitation study is recommended.
\end{abstract}

\section{Introduction}

Among the most common genetic abnormalities that can occur in the head areas are the stick-out protruding ears or prominent ears. The stick-out protruding ears are a condition that affects both sexes equally. The most common problems are the deformation anomaly of concha and the unformed antihelical fold od of the ear. The diagnosis of prominent ears is always subjective. Although various measurements have been made for the dimensions of the ears, the terms normal ears and dumbo ears are unclear. The incidence of stick out protruding ears is about $5 \%$ of the world's population, as it is the most common ear deformity. Otoplasty is one of the most common pediatric otolaryngological procedures. The signs of this deformity are mainly aesthetic and the corrective procedures are performed by a wide range of doctors, either in a private clinic, or by plastic surgeons or otolaryngologists and pediatricians. Surgical correction of the protruding ears is usually performed at the ages of 4 to 5 years, when the ear completes its development. There is no specific timetable for surgical or non-surgical implementation, however, the ultimate goal is to prevent children from being bullied at school. $[1,2,3]$ Today, the correction is covered by a non-invasive method through the product "Earbuddies" as it seems in the figure 1. [4] It should be noted that this method is based on the technique that a splint placed in the ear canal is fastened with tape. The pressure of the splint suspends the spring of the cartilage of the ear, while correcting its natural curves. When the cartilage hardens over time, a permanent and controlled shape is created. Both methods of otoplasty, invasive and non-invasive, present a number of problems that the invention is going to remedy. Specifically, there are both aesthetic and functional problems after completing the treatment. These include pain during sleep, increased sunburn, bleeding problems, and discomfort when patients wear glasses, a hat or hearing aids. The most basic disadvantage of all these methods, whether invasive or non-invasive, is the fact that after some time the ears come back into their initial place. [1, 2] Therefore, there is a clear need for a non-invasive method that can meet the needs of the patients. Namely, the presence of a product that does not require the intervention of an otolaryngologist or a plastic surgeon, but the role of the physician is only advisory. The creation of the case that fits into the ear will fill exactly this gap that exists today on the world market. The case can be used by people of all ages, since one or more cases are created for each human individually. All the necessary steps that were followed during experimental procedure for the study, design and construction of the artificial ear splint are illustrated bellow.

\footnotetext{
${ }^{*}$ Corresponding author: franki.thanasis@gmail.com
} 


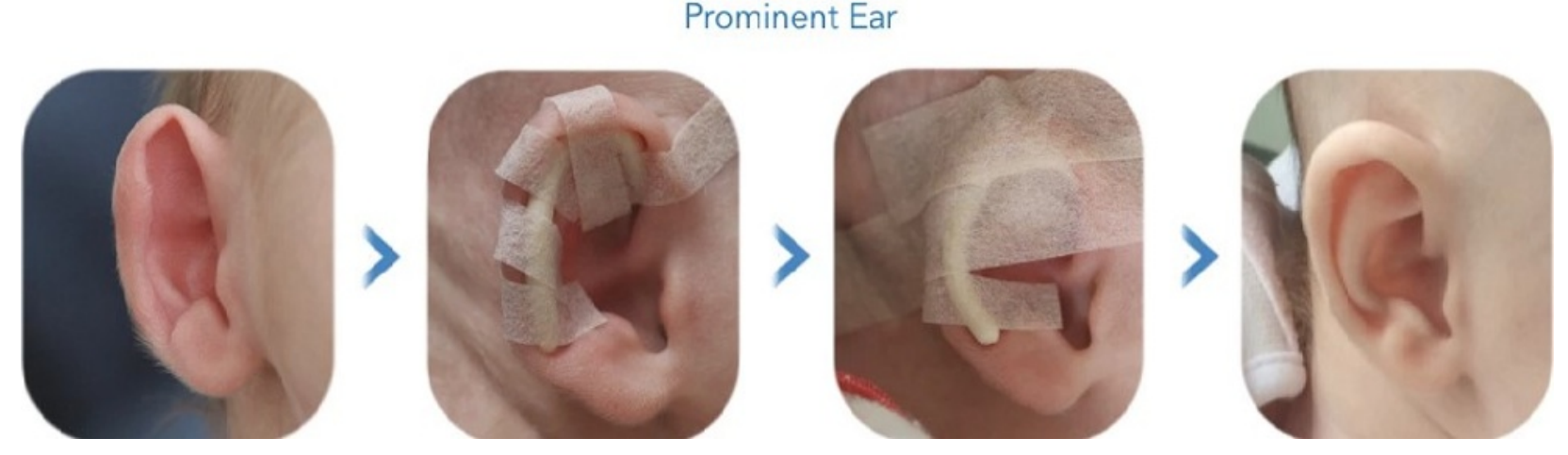

Fig. 1. Illustration of the product Earbuddies and how it corrects the deformity of the stick out protruding ears. [4]

\section{Methods}

\subsection{Design of the artificial ear splint model}

During the design of the artificial ear splint model a novel method was created. This model aims to restore stick-out ears back to their normal position, according to human anatomical features, through forces exerted on the helix and antihelix of the ear. The natural complexities of the ear surfaces presented the main challenge towards the success of this project. For this reason, a specific methodology had to be followed, involving photogrammetry technique, design and reverse engineering principles. Photogrammetry has emerged as a leading approach for photorealistic digital replication and 3D scanning of real-world objects, particularly in areas of cinematic visual effects and interactive entertainment. While the technique generally relies on simple photography methods, the foundational practices for the field of human photogrammetry remain relatively undocumented. Human subjects are significantly more complex than still life, both in terms of photogrammetric capture, and in digital reproduction. [5] Initially we had to found the sample of the human ear and one way to scan it. This was achieved by using the photogrammetry technique. First, we had to find the appropriate photographic instrument. A mobile phone with a 48 megapixel camera and multi-taking mode, was the medium of producing a range of photos of the same object from different angles. The purpose of many shots was to illustrate the final model with more details and eliminate the "noise" from it. This was followed by the selection of the space, as the lighting is the main factor for the taking of a photo. First, one hundred photos were taken outdoors with natural light and then one hundred photos were taken inside by using the camera's flash. The next step was to import all the photos into the Agisoft (Metashape) program. Agisoft is a software that performs photogrammetric processing of digital images of the ear and produced its three-dimensional digital model. The process was completed with one problem, the lack of scale from the digital model. [6] This problem was solved in the Meshlab program, an open source system that provides a set of tools for editing and defining project points. More specifically, 3 points were selected between the helix and the head, the actual distances were measured and then, by selecting the same 3 points within the program, the scale of the design was adjusted.

For display, data exchange, and construction, the 3D digital model was divided into a three-dimensional model consisting of 120 different vertices. However, the 3D representation can be modified and after numerical simulation it was no longer corresponds to the original model. Then required a method of reverse engineering in order to recreate a three dimensional model. [7, 8] The solution was provided by using Geomagic, a software that provides accurate three dimensional digital models and assemblies of CAD type natural objects. This software helped to modify the model file from .obj to $x_{-} t$ file. The .obj file format is a simple data format that represents the geometry of two-dimensional and three-dimensional designs, while the. $\mathrm{x}$ t file format indicates a Parasolid file that makes it possible to process model surfaces in a CAD program. The whole process is schematically described in Fig. 2. 


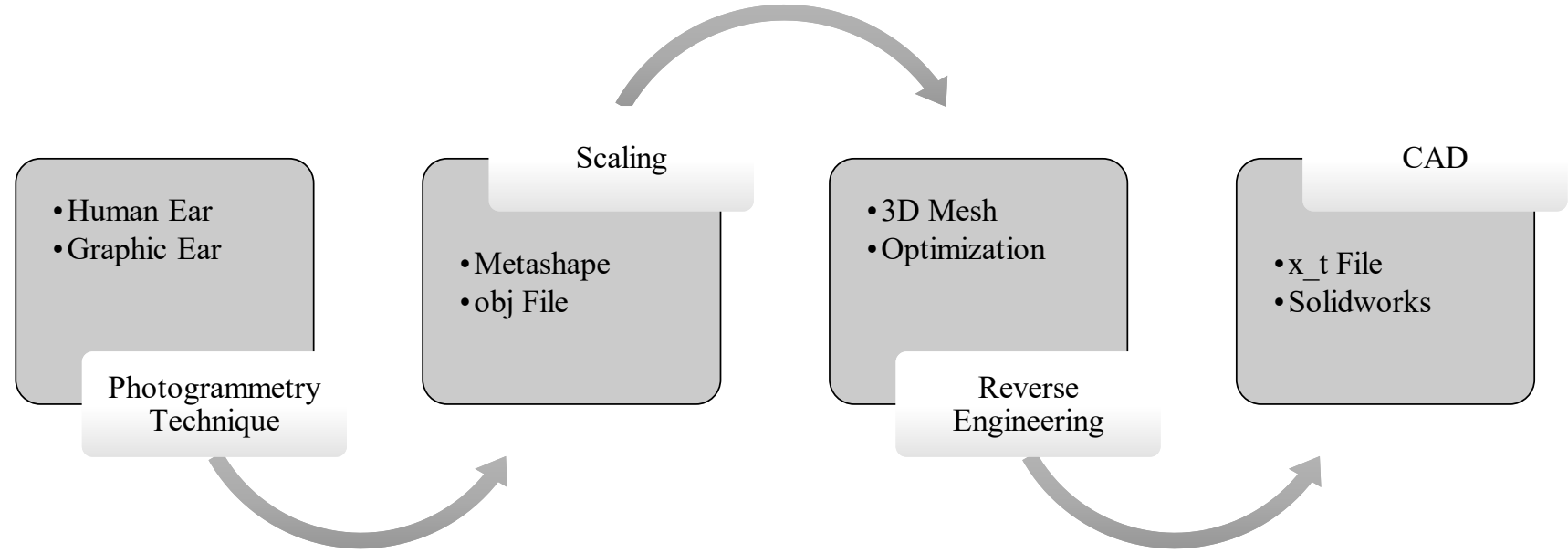

Fig. 2. Conversion process from Human ear to CAD model ear. The human ear is converted to graphic ear (obj file) through photogrammetry technique and after some modifications to 3D mesh, making tests and optimizations, the CAD model ear ( $\mathrm{x} \_\mathrm{t}$ file) is created.

\subsection{Production of the artificial ear splint model}

As for the design part, together with the 3D printing process, they are the two most difficult and most important parts of the artificial ear splint production process. In particular, the design was carried out in the Solidworks program and first step needed to be done, was to cut the outer material around the ear and then remove the inner material, leaving only specific surfaces. The shape that emerged from the procedure was also the final shape of the splint, with the only problem being the lack of scale, because the splint had to be applied to the ear. Therefore, after various tests and measurements, we ended up with an increase in thickness by $1,005 \mathrm{~mm}$. [9]

Next, the most important step in the preparation of the ear splint was the 3D printing method, filament deposition material (FDM). The M200 model of the Zortrax equipped with the V3 Hotend head was the 3D printer in which all the experiments were conducted. First of all, in the software provided by this company, Z-Suite, the necessary commands had to be set and it had to be decided which materials will be used. The experiments were performed with Z-ABS, while the basic material of the ear splint is Z-FLEX. At the same time, the first successful 3D printing attempt with Z-FLEX material on a M200 3D printer model was made, according to the company's literature, because the head was changed from V2 to V3 in order to print the Z-FLEX material.

Figure 3 shows the illustration of the design procedure for the artificial ear splint model that have been described before. In particular, it seems the whole methodology, how from a simple human ear, it can be fabricated, 3D printed an object that can offers a solution to everyday problems, such as the deformity of stick out protruding ears. Especially, the last picture shows the back view of a human who wears the ear splint.

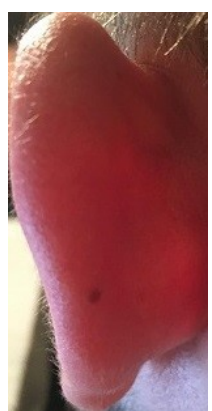

a)

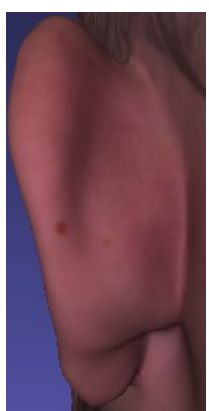

b)

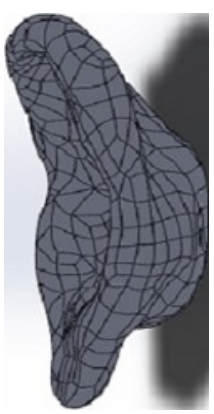

c)

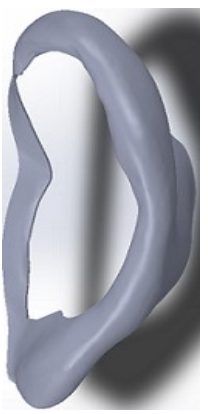

d)

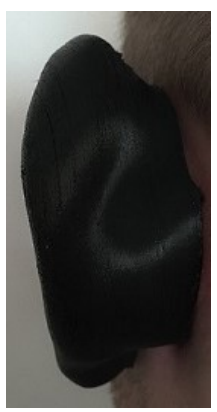

e)

Fig. 3. Illustration of the design procedure for the artificial ear splint model. The human ear (a) is used to give the digital model of the ear. (b) This shape is used as a basis for the computer aided design (CAD) model (c), which gives the final form (d), after some modifications, the artificial ear splint model is worn by a human (e). [6, 9] 


\section{Experiments}

\subsection{Experimental procedure}

As for the experimental process, it took only 16 grams of flex material, setting the appropriate settings on the program's platform, and after 4 hours and 43 minutes the ear splint was ready for use. Its dimensions according to the model of the human ear used are $79.36 \mathrm{~mm}$ long, $34.01 \mathrm{~mm}$ wide and $44.05 \mathrm{~mm}$ high just as it is applied to the ear. The commands set in the program are shown at table 1.

Table 1.3D printing settings for materials flex and abs.

\begin{tabular}{|c|c|c|}
\hline 3D Printing settings & Flex & Abs \\
\hline & & $0.4 \mathrm{~mm}$ \\
\hline Nozzle Diameter & $0.4 \mathrm{~mm}$ & $0.09 \mathrm{~mm}$ \\
\hline Layer Thickness & $0.09 \mathrm{~mm}$ & $230^{\circ} \mathrm{C}$ \\
\hline Extrusion Temperature & $230^{\circ} \mathrm{C}$ & $50{ }^{\circ} \mathrm{C}$ \\
\hline Platform & $60{ }^{\circ} \mathrm{C}$ & $90 \%$ \\
\hline Infill Density & $80 \%$ & \\
\hline
\end{tabular}

\subsection{Abs material}

The abs material is a flexible and economical material that perfectly suits the needs of users who are dealing with 3D printing for the first time and have one of the M200 or M200 Plus models of Zortrax. Its low cost allows for many effective and different tests across the available range of dimensions for the thickness of each layer. This material is characterized on the one hand by resistance to high temperatures, resistance to basic operating tests and on the other hand, by a high level of shrinkage. The problem with this material was that it did not meet the basic requirement of the splint, to give elasticity and therefore it was used exclusively for the study and correction the problems of the final ear splint design. Therefore, there was a need to find an elastomeric material that is human-friendly and provides the splint with the required elasticity of an object that fits right on a human ear. Figure 4 shows the ear splint model which was successfully fabricated with abs material on a $3 \mathrm{D}$ printer. [10]

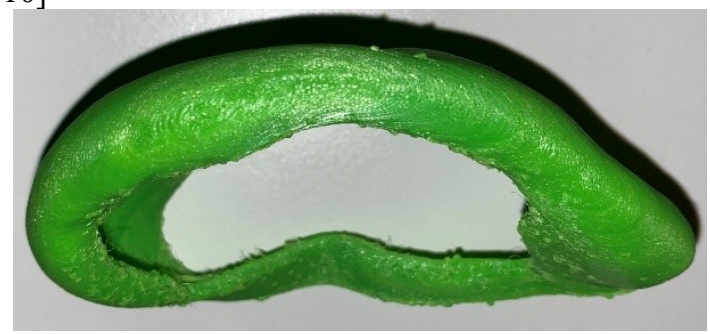

Fig. 4. Illustration of the artificial ear splint model, after successfully 3D printed with abs material.

\subsection{Flex material}

The flex material was the one who solved the problem. An elastomeric and thermoplastic material of the polyester category with a print speed of $45 \mathrm{~mm}$ per second $(\mathrm{mm} / \mathrm{s})$. The thread has a large adhesion between the layers and a tolerance of dimensions less than $+/-0.02 \mathrm{~mm}(\mathrm{~mm})$. Also, flex is elastic, can bend without breaking and is resistant to various chemicals such as gasoline, ethyl alcohol, butane and carbon monoxide. The only shade of this filament is black. Flex is ideal for 3D printing models that are intended to be folded frequently, such as tires and elastic hinges. Its elasticity, texture and the fact that it is human-friendly are the reasons that it was chosen as the basic material for printing the artificial ear splint. Figure 5 shows the ear splint model which was successfully fabricated with flex material on a 3D

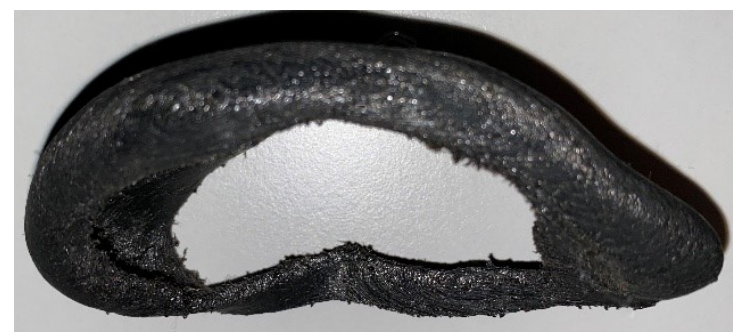

printer. [11]

Fig. 5. Illustration of the artificial ear splint model, after successfully $3 \mathrm{D}$ printed with flex material. 


\section{Results}

We presented a non-invasive method of restoring the stick out protruding ears by using an artificial ear splint model. The way it was designed and manufactured allows it to be applied to different types of people with prominent ears, regardless of age and geometry of the ear, since one or more cases are created for each human individually, using reverse engineering and 3D printing techniques. Applying the case to the human ear, any patient could gain the substantial benefits of its usage. On the one hand, it is very lightweight, since it weighs only a few grams, and on the other hand, it provides comfort and convenience to the wearer, while the other already existing methods are causing pain and other functional problems. Even during sleep when the head is pressed against the pillow no pain or discomfort has been observed. The use of an elastomeric and thermoplastic material, namely flex, has contributed to the aforementioned results. Having been used to fabricate the ear splint, this material has been proven to give it the flexibility it requires/is necessary for such an object being placed on the head. The tray model was printed on a 3D printer, specifically the Zortrax 3D printer M200 model. The V3 head was added in order to print the Z-FLEX material. The invention can be manufactured on any 3D printer model that allows. It is worth mentioning that there was no abrasion, itching or pain at any point during the ear splint application. Meanwhile, it should be emphasized that it is possible to use either myopic glasses or sunglasses over the case.

As for the manufacturing process, there are some modifications that could be made. For example, a different way of approaching the sample, which includes scanning from a modern 3D scan with the ability to capture the object at an angle of 180 degrees. In this way the human factor is removed from the process and the possibility of an error occurring, which in the case of being studied, contributed to the taking of the photographs of the ear model. Also, there could be used arms that hold the camera in place and provide the ability to take photos from all possible angles and perspectives.

\section{Discussion}

The creation of the splint was carried out at an initial level in the context of the preparation of an undergraduate dissertation, and a laboratory product was prepared. However, many future improvements that can be made in the near future have been discussed and observed, evolving the final product. Initially, it is helpful to conduct a series of experiments with the flex filament as a basic material to prove that it is human-friendly, although in the literature does not mention the opposite. In addition, a future proposal for Zortrax is the preparation of the filament from transparent material (Natural Transparency), as the only available shade for the flex filament at the moment, creates an aesthetically strange result.

Next, it should be noted that without measuring the tension exerted by the ear splint, since no specialized measuring instruments were available, it was observed that force applied on the helix and antihelical fold of the ear. Therefore, there is a need for further research and study of the method with the assistance of specialized scientists, such as otolaryngologists, invasive surgeons, etc. This research can be carried out in the context of a clinical rehabilitation study such as the term in medical science, in order to obtain experimental results on the practicality of the artificial splint. While after the completion of the clinical study and the taking of a sample, the modeling of the object is proposed, with the aim of its possible subsequent industrial production.

This project is supported by the "Athena Research and Innovation Center- Institute of Language and Speech Processing (ILSP)".

\section{References}

1. A. L. Schneider, D. M. Sidle, Department of Otolaryngology-Head and Neck Surgery 26, 19-29 (2018)

2. A. Gantous, A. J. Tasman, J. C. Neves, Facial Plast Surg Clin North Am 26, 181-192 (2018)

3. L. Kajosaari, J. Pennanen, T. Klockars, Int J Pediatr Otorhinolaryngol 100, 52-56 (2017)

4. www.earbuddies.com

5. T. Boe, C. Carter, International Journal of Computer Graphics \& Animation 10, (2020)

6. www.agisoft.com

7. R. Bénière, G. Gesquiere, F. Breton, W. Puech, G. Subsol, Computer-Aided Design 45, 1382-1393 (2013)

8. S. Gauthier, W. Puech, R. Bénière, G. Subsol, LIRMM Laboratory 93, 67-83 (2017)

9. $\quad$ www.Solidworks.com, S.N: 9020008545019225 RTC2 22H7, (2019) 
10. www.zortrax.com/filaments/z-abs/

11. https://zortrax.com/filaments/z-flex/ 